\title{
Proteomic database search engine for two-dimensional partial covariance mass
}

\section{spectrometry}

Taran Driver, ${ }^{\dagger}$ Nikhil Bachhawat, ${ }^{\dagger}$ Rüdiger Pipkorn, ${ }^{\ddagger}$ Leszek J. Frasiński, ${ }^{\dagger}$ Jon P. Marangos ${ }^{\dagger}$ Marina Edelson-Averbukh ${ }^{\dagger}$ and Vitali Averbukh $^{*, \dagger}$

$\dagger$ The Blackett Laboratory, Imperial College London, Prince Consort Road, London SW7 2AZ, United Kingdom

$\ddagger$ German Cancer Research Centre, Department of Translational Immunology, Im Neuenheimer Feld 580, 69120 Heidelberg, Germany

E-mail: vv.averbukh@imperial.ac.uk

\section{Supporting Information Available}

Containing:

- Peak integration and peak score in 2D-PC-MS

- 1D MS/MS search engine results

\section{Peak integration and peak score in 2D-PC-MS}

The volumes of the 2D-PC-MS peaks, $V^{(n)}(X, Y$, TIC) of Eqs. $(2,3)$, were calculated using a bivariate spline integration routine from the SciPy scientific computing library. The 
area considered for integration was separately defined for each correlation peak to account for changing shape due to different charge state/isotopic distribution. The integration domain was chosen as a polygon with corners defined by 100 rays (intentionally over-sampled) emanating from the local maximum of the correlation peak. The length of each ray was determined as $\min (\mathrm{x}, \mathrm{y})$, where $\mathrm{x}$ is the length at which a lower threshold is reached (0 for our measurements) and y is a pre-defined maximum length in m/z bins (15 for our measurements).

The correlation score of Eq. (2) is used as a global parameter for picking the true correlations across the 2D-PC-MS map. It is of interest to check whether its absolute value bears a significance. The results shown in Fig. S1 imply that this is indeed the case. There appears to exist a certain threshold value of the correlation score above which the scored correlations are the true ones, and this threshold value (around 300) is weakly independent on the charge state and the sequence of the peptides in our measurements . Of course, the correlation score threshold presumably depends on the instrument and the $\mathrm{m} / \mathrm{z}$ measurement parameters.

\section{D MS/MS search engine results}

The Mascot search for the 50/50 mixture of the reverse sequence peptides, GSNKGAIIGLM and MLGIIAGKNSG was performed using a non-selective digest of the UniProtKB/SwissProt database, with precursor $\mathrm{m} / \mathrm{z}$ tolerance $5 \mathrm{ppm}$ and fragment ion $\mathrm{m} / \mathrm{z}$ tolerance 0.8 Da. No modifications were specified. The correct database sequence was ranked second or fourth by the Mascot search depending on the number of microscans used, see Fig. S2.

Another MS/MS search engine, MS-GF+, applied to the same data as above produced an incorrect identification, see Fig. S3.

Also the database search module of PEAKS failed for the case of the reverse sequence mixture. Within this algorithm, one first looks for a series of plausible sequences de novo and only then continues with the database inquiry step. The single sequence found by PEAKS 


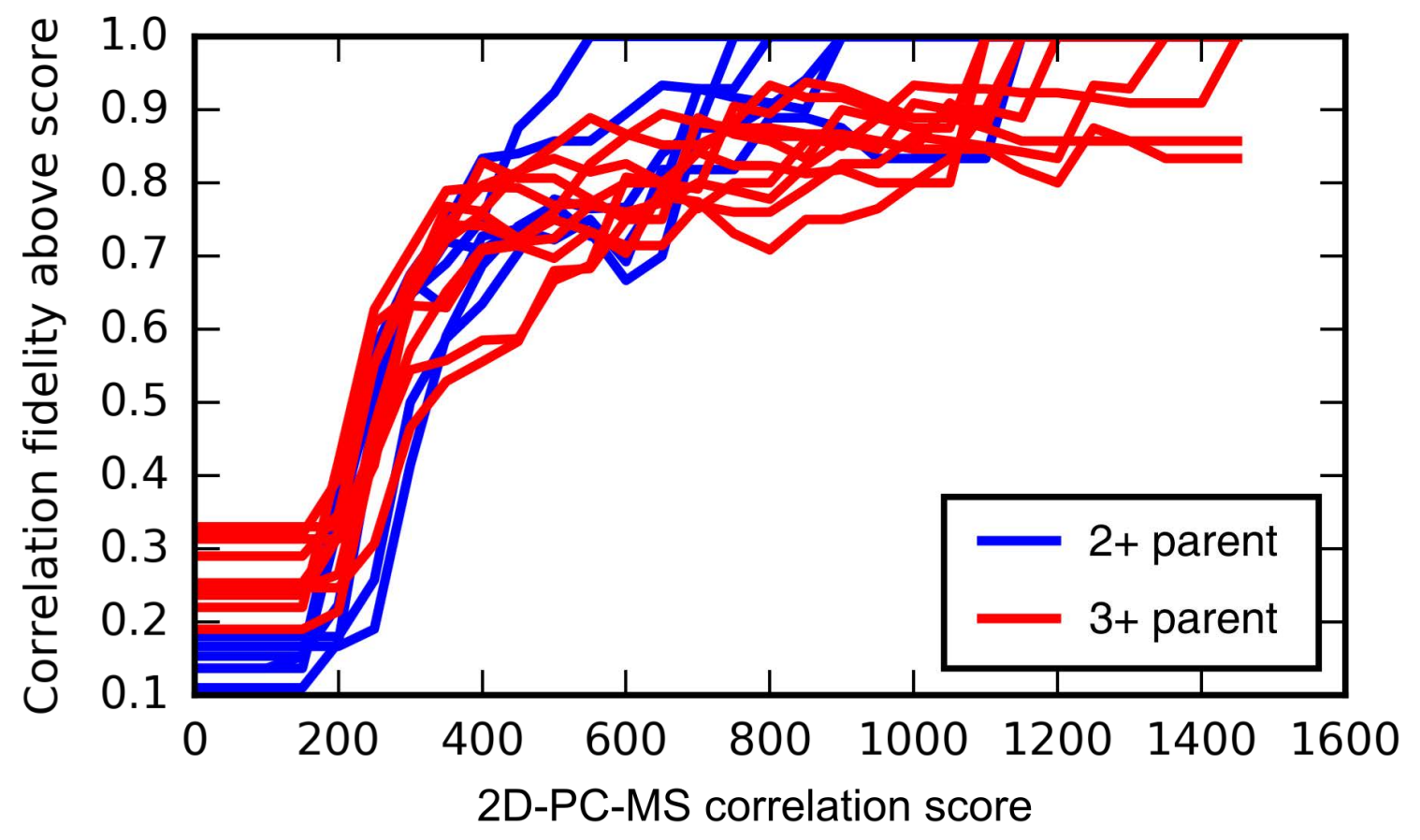

Figure S1: The fraction of 2D-PC-MS correlation features identified as canonical fragment ion correlations by the automated correlation matching procedure ("Correlation fidelity", y-axis) is shown for all correlations scoring above a particular 2D-PC-MS correlation score (x-axis) for the following $2+$ (blue) and $3+$ (red) parent ions: $[\mathrm{MLGIIAGKNSG}+2 \mathrm{H}]^{2+},[\mathrm{MLGIIAGKNSG}+2 \mathrm{H}]^{3+},[\text { GSNKGAIIGLM+2H }]^{2+}$, $\left[\right.$ GK $\left(\right.$ Ac)GGKGLGK $(\text { Ac)GGAKR }+2 \mathrm{H}]^{2+},[\text { GK(Ac)GGKGLGK(Ac)GGAKR+3H }]^{3+}$, $[\operatorname{VTIMPK}(\mathrm{Ac}) \mathrm{DIQLAR}+2 \mathrm{H}]^{2+},[\mathrm{VTIMPK}(\mathrm{Ac}) \mathrm{DIQLAR}+3 \mathrm{H}]^{3+}$, $\left[\mathrm{LGEY}(\text { nitro)GFQNAILVR }+2 \mathrm{H}]^{2+},\left[\mathrm{LGEY}(\text { nitro)GFQNAILVR }+3 \mathrm{H}]^{3+}\right.\right.$, $[\text { VTIMPKDIQLAR+2H }]^{2+},[\text { VTIMPKDIQLAR }+3 \mathrm{H}]^{3+},[\text { GGNFSGR(Me)GGFGGSR }+3 \mathrm{H}]^{3+}$, $\left[\mathrm{GWGR}\left(\mathrm{Me}_{2}\right) \text { EENLFSWK }+3 \mathrm{H}\right]^{3+},\left[\text { EQFDDpYGHMRF-NH }{ }_{2}+3 \mathrm{H}\right]^{3+}$,

[VTIMPK $\left.\left(\mathrm{Me}_{3}\right) \mathrm{DIQLAR}+3 \mathrm{H}\right]^{3+}$. The threshold value of the correlation score above which the true correlation signals lie $(S \approx 300)$ is weakly dependent of the sequence and the charge state. 
$3 \mu \mathrm{s}, 10$ scans

\begin{tabular}{|l|c|c|l|}
\hline Score & Mr(calc) & Delta & \multicolumn{1}{|c|}{ Sequence } \\
\hline 30.6 & 1059.5787 & -0.0030 & MIGLAWLLSG \\
\hline 28.9 & 1059.5746 & 0.0010 & GSNKGAIIGLM \\
\hline 25.1 & 1059.5746 & 0.0010 & DTCIINRI \\
\hline 25.1 & 1059.5746 & 0.0010 & ESCLRNLLL \\
\hline 25.0 & 1059.5713 & 0.0044 & SNGKGAIIGPF \\
\hline 24.1 & 1059.5746 & 0.0010 & CSEAVRVALL \\
\hline 23.9 & 1059.5787 & -0.0030 & EIEWALGLM \\
\hline 22.9 & 1059.5787 & -0.0030 & MIGLTHYLI \\
\hline 21.7 & 1059.5787 & -0.0031 & GFPIIGVGGIM \\
\hline 21.3 & 1059.5787 & -0.0030 & SMTLHFVIL \\
\hline
\end{tabular}

$3 \mu \mathrm{s}, 40$ scans

\begin{tabular}{|l|c|c|l|}
\hline Score & Mr(calc) & Delta & \multicolumn{1}{|c|}{ Sequence } \\
\hline 30.7 & 1059.5787 & -0.0030 & MIGLAWLLSG \\
\hline 28.9 & 1059.5746 & 0.0010 & GSNGGAIIGLM \\
\hline 26.5 & 1059.5747 & 0.0010 & ISDVRGMLGL \\
\hline 26.3 & 1059.5713 & 0.0044 & SNGKGAIIGP \\
\hline 25.2 & 1059.5746 & 0.0010 & DTCIINRLI \\
\hline 25.2 & 1059.5746 & 0.0010 & ESCLRNLLL \\
\hline 24.1 & 1059.5746 & 0.0010 & CSEAVRVALL \\
\hline 23.0 & 1059.5787 & -0.0030 & MIGLTHYLI \\
\hline 22.9 & 1059.5787 & -0.0030 & KIEWALGLM \\
\hline 22.6 & 1059.5787 & -0.0030 & $\underline{\text { MLGLWSAIVA }}$ \\
\hline
\end{tabular}

$3 \mu \mathrm{s}, 20$ scans

\begin{tabular}{|l|c|c|l|}
\hline Score & Mr(calc) & Delta & \multicolumn{1}{|c|}{ Sequence } \\
\hline 30.7 & 1059.5787 & -0.0030 & MIGLAWLLSG \\
\hline 28.9 & 1059.5746 & 0.0010 & GSNGGAIIGLM \\
\hline 25.2 & 1059.5746 & 0.0010 & DTCIINLI \\
\hline 25.2 & 1059.5746 & 0.0010 & ESCLRNLLL \\
\hline 25.0 & 1059.5713 & 0.0044 & SNGKGAIIGPF \\
\hline 24.1 & 1059.5746 & 0.0010 & CSEAVRVALL \\
\hline 23.0 & 1059.5787 & -0.0030 & MIGLTHYLI \\
\hline 22.1 & 1059.5787 & -0.0030 & LGLSWAGMLL \\
\hline 21.8 & 1059.5787 & -0.0031 & GFPIIGVGGIM \\
\hline 21.4 & 1059.5747 & 0.0010 & MLGRLIDVSG \\
\hline
\end{tabular}

$3 \mu \mathrm{s}, 50$ scans

\begin{tabular}{|l|c|c|l|}
\hline Score & Mr(calc) & Delta & Sequence \\
\hline 30.7 & 1059.5787 & -0.0030 & MIGLAWLLSG \\
\hline 28.9 & 1059.5746 & 0.0010 & GSNKGAIIGLM \\
\hline 26.5 & 1059.5747 & 0.0010 & ISDVRGMLGL \\
\hline 25.2 & 1059.5746 & 0.0010 & DTCIINRLI \\
\hline 25.2 & 1059.5746 & 0.0010 & ESCLRNLLL \\
\hline 25.1 & 1059.5713 & 0.0044 & SNGKGAIIGPF \\
\hline 24.1 & 1059.5746 & 0.0010 & CSEAVRVALL \\
\hline 23.0 & 1059.5787 & -0.0030 & MIGLTHYLI \\
\hline 22.6 & 1059.5787 & -0.0030 & MLGLWSAIVA \\
\hline 22.1 & 1059.5787 & -0.0030 & $\underline{\text { LGLSWAGMLL }}$ \\
\hline
\end{tabular}

$3 \mu \mathrm{s}, 30$ scans

\begin{tabular}{|l|c|c|l|}
\hline Score & $\mathbf{M r}($ calc) & Delta & \multicolumn{1}{|c|}{ Sequence } \\
\hline 30.7 & 1059.5787 & -0.0030 & MIGLAWLLSG \\
\hline 27.7 & 1059.5746 & 0.0010 & GSNKGAIIGLM \\
\hline 25.2 & 1059.5746 & 0.0010 & DTCIINRLI \\
\hline 25.2 & 1059.5746 & 0.0010 & ESCLRNLLL \\
\hline 24.9 & 1059.5713 & 0.0044 & $\underline{\text { SNGKGAIGPF }}$ \\
\hline 24.1 & 1059.5746 & 0.0010 & $\underline{\text { CSEAVRVALL }}$ \\
\hline 23.0 & 1059.5787 & -0.0030 & MIGLTHYLI \\
\hline 22.9 & 1059.5787 & -0.0030 & $\underline{\text { KIEWALGLM }}$ \\
\hline 22.1 & 1059.5787 & -0.0030 & $\underline{\text { LGLSWAGMLL }}$ \\
\hline 21.8 & 1059.5787 & -0.0031 & $\underline{\text { GFPIGVGGIM }}$ \\
\hline
\end{tabular}

$5000 \mu \mathrm{s}, 1$ scan

\begin{tabular}{|l|c|c|c|}
\hline Score & Mr(calc) & Delta & Sequence \\
\hline 30.4 & 1059.5787 & -0.0030 & MIGLAWLLSG \\
\hline 29.3 & 1059.5787 & -0.0030 & $\underline{\text { MLGLWSAIVA }}$ \\
\hline 26.3 & 1059.5747 & 0.0010 & $\underline{\text { ISDVRGMLGL }}$ \\
\hline 25.5 & 1059.5746 & 0.0010 & GSNKGAIIGLM \\
\hline 24.8 & 1059.5746 & 0.0010 & $\underline{\text { DTCIINRLI }}$ \\
\hline 24.8 & 1059.5746 & 0.0010 & $\underline{\text { ESCLRNLLL }}$ \\
\hline 23.0 & 1059.5787 & -0.0030 & $\underline{\text { LGLSWAGMLL }}$ \\
\hline 22.8 & 1059.5787 & -0.0030 & $\underline{\text { MIGLTHYLI }}$ \\
\hline 21.5 & 1059.5746 & 0.0010 & NAGKEKGILM \\
\hline 21.5 & 1059.5787 & -0.0031 & $\underline{\text { GFPIIGVGGIM }}$ \\
\hline
\end{tabular}

Figure S2: Results of the Mascot database search (peptide score) for the 50/50 mixture of the reverse sequence peptides, GSNKGAIIGLM and MLGIIAGKNSG. The number of scans and the number of microscans within each scan are designated for each search result. The correct sequence, highlighted by red frame, is not identified by Mascot as the top scoring one for any of the searches.

\begin{tabular}{|l|l|}
\hline \multicolumn{2}{|l|}{ MS-GF+ output } \\
\hline ID sequence & MIGLTHYLI \\
\hline MS-GF Raw Score & 33 \\
\hline MS-GF Spec E Value & $1.4526 \mathrm{e}-7$ \\
\hline MS-GF E Value & 16.2568 \\
\hline
\end{tabular}

Figure S3: Results of the MS-GF+ database search for the 50/50 mixture of the reverse sequence peptides, GSNKGAIIGLM and MLGIIAGKNSG. MS/MS data were averaged over 50 scans, 3 microscans each. The identified sequence produced by MS-GF + is incorrect and is one of the top-scoring Mascot sequences. 
at the de novo step was YVGLNVGGGLL with the score of 21 that was insufficient to pass to the database inquiry, see Fig. $\mathrm{S} 4$.

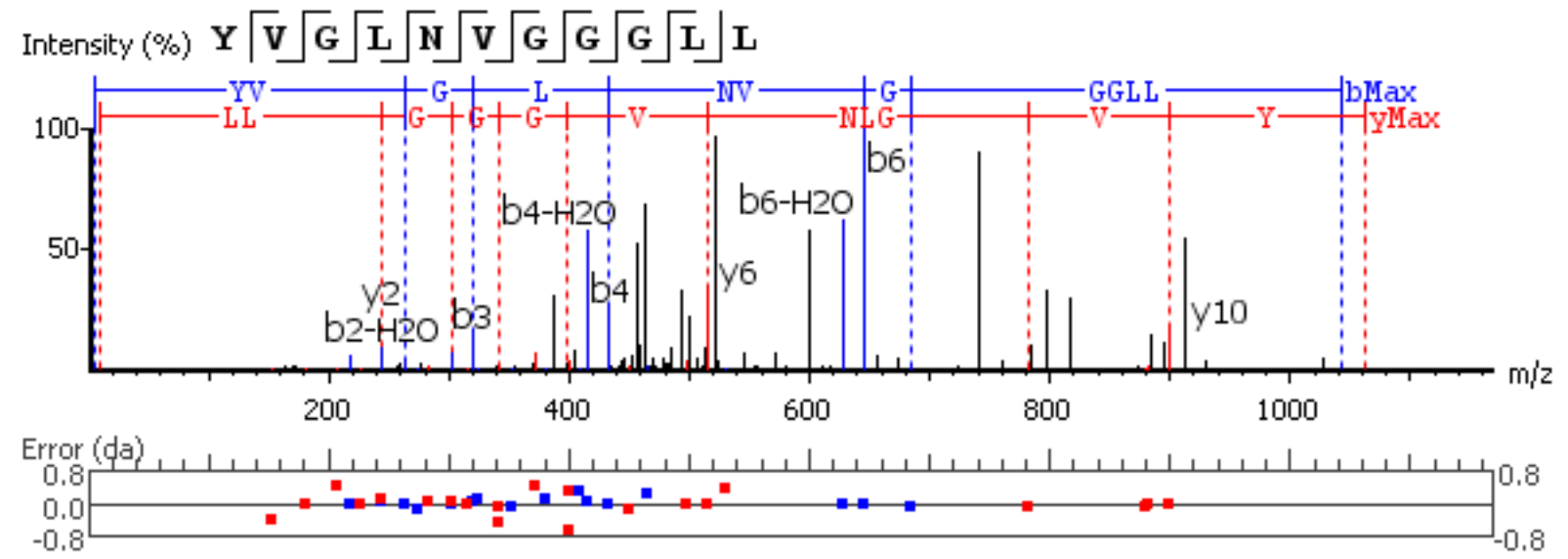

\begin{tabular}{|c|c|c|c|c|c|c|c|c|c|c|}
\hline \multicolumn{11}{|c|}{ Ion Match } \\
\hline$\#$ & $b$ & $\mathrm{~b}-\mathrm{H} 2 \mathrm{O}$ & b-NH3 & $b(2+)$ & Seq & $y$ & $y-\mathrm{H} 2 \mathrm{O}$ & $y-\mathrm{NH} 3$ & $y(2+)$ & \# \\
\hline 1 & 164.07 & 146.06 & 147.04 & 82.54 & $Y$ & & & & & 11 \\
\hline 2 & 263.15 & 245.02 & 246.11 & 132.07 & V & 898.55 & 880.60 & 881.51 & 449.90 & 10 \\
\hline 3 & 320.09 & 302.13 & 303.13 & 160.58 & G & 799.47 & 781.54 & 782.44 & 399.88 & 9 \\
\hline 4 & 433.24 & 415.14 & 416.22 & 217.09 & $\mathrm{~L}$ & 742.45 & 724.44 & 725.42 & 371.27 & 8 \\
\hline 5 & 547.29 & 529.28 & 530.26 & 274.33 & $\mathrm{~N}$ & 629.36 & 611.35 & 612.33 & 315.23 & 7 \\
\hline 6 & 646.40 & 628.39 & 629.33 & 323.55 & V & 515.30 & 497.33 & 498.29 & 258.16 & 6 \\
\hline 7 & 703.38 & 685.44 & 686.35 & 352.26 & G & 416.25 & 398.24 & 399.88 & 208.15 & 5 \\
\hline 8 & 760.40 & 742.39 & 743.37 & 380.55 & G & 359.23 & 341.30 & 342.71 & 180.11 & 4 \\
\hline 9 & 817.42 & 799.41 & 800.39 & 408.84 & G & 302.13 & 284.08 & 285.18 & 152.00 & 3 \\
\hline 10 & 930.50 & 912.49 & 913.48 & 465.46 & L & 245.02 & 227.16 & 228.16 & 123.09 & 2 \\
\hline 11 & & & & & L & 132.10 & 114.09 & 115.07 & 66.55 & 1 \\
\hline
\end{tabular}

Figure S4: Results of the PEAKS database search for the 50/50 mixture of the reverse sequence peptides, GSNKGAIIGLM and MLGIIAGKNSG. MS/MS data were averaged over 50 scans, 3 microscans each. Only a single (incorrect) sequence could be determined at the preliminary de novo step, precluding the subsequent database inquiry.

ProLuCID (using an improved SEQUEST algorithm) does manage to identify the correct sequence as a top hit with Z-score of about 4.37 see Fig. S5, which according to the authors is the preferred criterion for the LTQ data analysis. The Z-score value of the correct sequence corresponds to false-positive rate of about $10 \%$. 


\begin{tabular}{|l|l|l|l|l|l|}
\hline \multicolumn{5}{|l|}{ ProLuCID results } \\
\hline $\begin{array}{l}\text { Rank by } \\
\text { Xcorr }\end{array}$ & $\begin{array}{l}\text { Rank by } \\
\mathbf{S}_{\mathbf{p}}\end{array}$ & $\begin{array}{l}\text { Xcorr } \\
\text { score }\end{array}$ & Z score & $\begin{array}{l}\text { \# matched } \\
\text { ions }\end{array}$ & Sequence \\
\hline 1 & 10 & 1.6179 & 4.373734054 & 16 & V.GSNKGAIIGLM.V \\
\hline 2 & 8 & 1.4614 & 3.813613554 & 17 & V.MIGLVIGTEAG.Y \\
\hline 3 & 8 & 1.451 & 3.776282133 & 17 & A.YILGAKGILL.S \\
\hline 4 & 8 & 1.3916 & 3.563888612 & 17 & T.YLEPAANGII.P \\
\hline 5 & 8 & 1.2833 & 3.176080738 & 17 & M.KDGIGKGKTR.E \\
\hline 102 & 1 & 0.4364 & 0.144993564 & 24 & S.AAGGGGGGGGGARR.L \\
\hline
\end{tabular}

Figure S5: Results of the ProLuCID database search for the 50/50 mixture of the reverse sequence peptides, GSNKGAIIGLM and MLGIIAGKNSG. MS/MS data were averaged over 50 scans, 3 microscans each. The top scoring candidate is the correct sequence, with Z-score $\approx 4.37$ corresponding to $\approx 10 \%$ false-positive rate. 